\title{
An evaluation of the 25 by 25 goal for premature cardiovascular disease mortality in Taiwan: an age- period-cohort analysis, population attributable fraction and national population-based study
}

\author{
Shih-Yung Su, ${ }^{1}$ Wen-Chung Lee, ${ }^{1,2}$ Tzu-Ting Chen, ${ }^{1}$ Hao-Chien Wang, ${ }^{3,4}$ Ta-Chen Su, ${ }^{5,6}$ \\ Jiann-Shing Jeng, ${ }^{7}$ Yu-Kang Tu, ${ }^{1}$ Shu-Fen Liao, ${ }^{1,2}$ Tzu-Pin Lu, ${ }^{1}$ Kuo-Liong Chien ${ }^{1,8}$
}

- Additional material is published online only. To view please visit the journal online (http://dx.doi.org/10.1136/ heartasia-2017-010905).

For numbered affiliations see end of article.

\section{Correspondence to}

Dr Kuo-Liong Chien, Institute of Epidemiology \& Preventive Medicine, College of Public Health, National Taiwan University, Rm. 536, No. 17, Xuzhou Rd, Taipei 100, Taiwan; klchien@ntu.edu.tw

Received 24 March 2017 Revised 7 June 2017 Accepted 8 June 2017
CrossMark

To cite: Su S-Y, Lee W-C, Chen T-T, et al. Heart Asia 2017:9:1-9.. doi:10.1136/ heartasia-2017-010905

\section{ABSTRACT}

Objectives The aim of the 25 by 25 goal is to reduce mortality from premature non-communicable diseases by $25 \%$ before 2025 . Studies have evaluated the 25 by 25 goal in many countries, but not in Taiwan. The aim of this study was to estimate the 25 by 25 goal for premature mortality from cardiovascular diseases in Taiwan. Methods We applied the age-period-cohort model to project the incidence of premature death from cardiovascular disease from 2015 to 2024 and used the population attributable fraction to estimate the contributions of targeted risk factors. The probability of death was used to estimate the percent change. Results The percent change in business-as-usual trend during 2010-2024 was only a 6\% (range 1.7-10.7\%) lower risk of premature mortality from cardiovascular disease among men. The greatest reduction in the risk of mortality occurred with a $30 \%$ reduction in the prevalence of smoking; however, there was only a $14.5 \%$ (10.6-18.3\%) decrease in percent change and in the corresponding number of men (3706: range 3543-3868) who were prevented from dying. More than a $25 \%$ reduction in the percent change of premature cardiovascular disease mortality among women was achieved without control of any risk factor. To reach a 25\% reduction in men before 2025 , there needs to be a $70 \%$ reduction in the prevalence of smoking to reduce mortality by $26.2 \%$ (22.9-29.3\%).

Conclusions Cigarette smoking is the primary target in the prevention of cardiovascular disease. Through the stringent control of smoking, the goal of a $25 \%$ reduction in premature mortality from cardiovascular disease may be achieved before 2025 in Taiwan.

\section{INTRODUCTION}

Non-communicable diseases (NCDs) have become an issue in global health, resulting in health and economic burdens in the 21st century. More than 36 million people die from NCDs every year; almost half of the deaths from NCDs occur between the ages of 30 and 70 years, the so called 'premature' deaths, and almost three quarters of NCD deaths occur in low and middle income countries. ${ }^{1}$ The four main types of NCDs are cardiovascular disease, cancer, chronic respiratory disease, and diabetes. ${ }^{2}$ Among these, cardiovascular disease is the primary cause of premature deaths and accounts for almost half of all deaths from NCDs. ${ }^{3}$
Many health programmes have addressed the prevention and treatment of NCDs over the past decade. In 2011, the United Nations (UN) General Assembly promulgated a political declaration and asked their member states to achieve the goal of a $25 \%$ reduction in premature mortality due to NCDs before 2025 (abbreviated as the ' 25 by 25 goal'). ${ }^{4}$ In response to the 25 by 25 goal, WHO not only developed a global monitoring framework but also published a 2013-2020 Action Plan with several targets, such as the control of alcohol, physical inactivity, salt/sodium, tobacco, high blood pressure and diabetes/obesity, as well as improvements in medical treatment. ${ }^{5}$ In 2013, the World Heart Federation (WHF) proposed a Global Cardiovascular Disease Taskforce with the specific goal of achieving the 25 by 25 goal, but with a focus on only cardiovascular disease by addressing the major risk factors of tobacco use and hypertension. ${ }^{6}$

In response to these health initiatives, studies have evaluated the 25 by 25 goal in many countries but none has addressed the risk factors, especially those for cardiovascular disease, in Taiwan. ${ }^{7-15}$ Among the 10 leading causes of death in Taiwan in 2015, cardiovascular diseases, including heart disease and cerebrovascular disease, were the second and third leading causes of death ${ }^{16} ; 19 \%$ of the total deaths were caused by cardiovascular disease, and $44 \%$ of these were premature deaths. ${ }^{16}$

The aims of this study were to project the premature cardiovascular disease mortality rates to 2025 by age, period, and cohort analysis, and to estimate the 25 by 25 goal in Taiwan in order to answer the critical questions as to how many people could be saved from death by achieving the targets for risk factors, and how much the prevalence of risk factor targets needed to change in order to reach the 25 by 25 goal.

\section{METHODS}

\section{Data sources}

Records of premature deaths due to cardiovascular diseases from 1990 to 2014 in Taiwan were abstracted from the database provided by the Ministry of Health and Welfare and categorised into eight age groups $(30-34,35-39,40-44,45-49$, $50-54,55-59,60-64,65-69)$ and five period groups (1990-1994, 1995-1999, 2000-2004, 2005-2009, 2010-2014). Only heart disease and cerebrovascular disease were selected for this study 
according to the 9 th and 10 th versions of the International Classification of Diseases (ICD-9 from 1990 to 2008; and ICD-10 after 2009) codes for heart disease: 391, 392.0, 393-398, 410-414, 415.19, 416, and 420-429 (ICD-9), and I01-I02.0, I05-I09, I20-I25, I27, and I30-I52 (ICD-10). For cerebrovascular disease, the codes 430-438 and I60-I69 (for both ICD-9 and ICD-10) were selected. Population numbers from 1990 to 2014 were abstracted from the online database provided by the Department of Statistics of the Ministry of the Interior in Taiwan. Age and calendar year were similarly categorised. Data for the age-specific prevalence of hypertension and diabetes were taken from the database, Taiwan Behavioural Risk Factor Surveillance System, provided by the Health Promotion Administration in Taiwan. A total of 34406 hypertensive and 57574 diabetic premature deaths from 2010 to 2014 were identified. Data for the age-specific prevalence of hyperlipidaemia were taken from the database, National Health Interview Survey, provided by the Health Promotion Administration in Taiwan. A total of 21940 premature deaths in 2009 were identified. Data for the age-specific prevalence of smoking were taken from the database, Taiwanese Adult Smoking Behaviour Surveillance System, provided by the Health Promotion Administration in Taiwan. A total of 84171 premature deaths from 2010 to 2014 were available for analysis. The age-specific prevalence of smoking, diabetes, hypertension and hyperlipidaemia are presented in the online supplementary table S1.
The relative risks of high blood pressure, high total cholesterol and high blood glucose for cardiovascular disease were used to determine the conservative estimates for hypertension, hyperlipidaemia and diabetes, and were taken from the study by Singh and colleagues. ${ }^{17}$ The relative risk for smoking in cardiovascular disease was taken from the study by Kontis and colleagues. ${ }^{14}$ The relative risks of smoking, high blood pressure, high total cholesterol and high blood glucose are presented in the online supplementary table S2. We excluded body mass index (BMI) in our study design because of its potential invalidity in diagnosing obesity in Asians. The association between BMI and obesity in Asians had shown that those with a lower BMI were fatter than comparable whites. ${ }^{18}$ Previous studies have also indicated that the accuracy of BMI in predicting overweight or obesity is limited. ${ }^{19} 20$

\section{Statistical analysis}

The world standard (WHO 2000) population proportions were used to calculate the age-adjusted mortality rates. ${ }^{21}$ The age-period-cohort (APC) method proposed by Tzeng and Lee was used to forecast cardiovascular mortality. ${ }^{22}$ The non-identifiability problem (of parameter estimation) arises because the three temporal variables used in the APC model have an exact linear relationship: cohort+age=period; that is, given a person's birth year and his/her age of death, the calendar year in which the person died is then known.

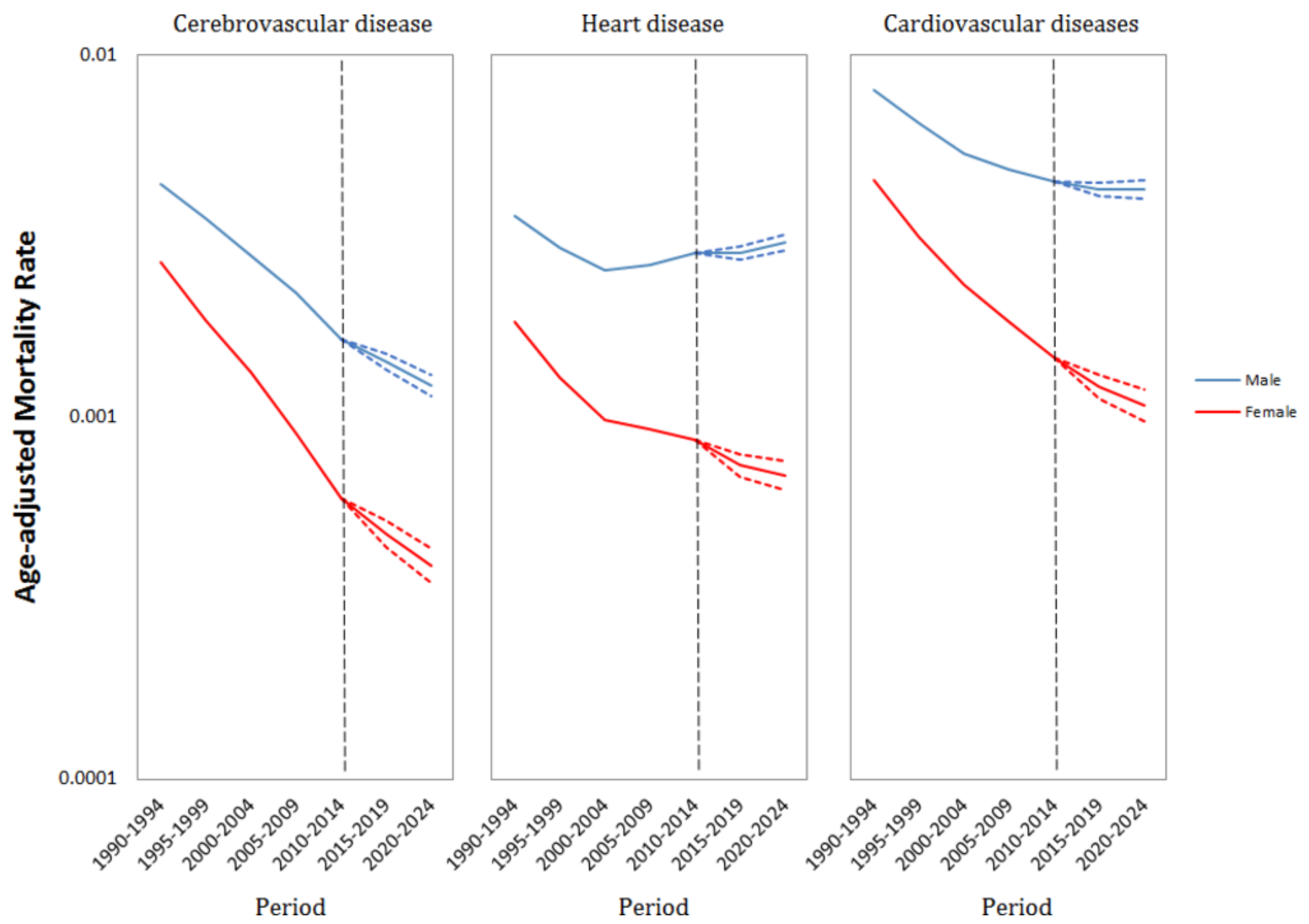

Figure 1 Age-adjusted mortality rates and confidence intervals (dotted line) for cerebrovascular disease, heart disease and cardiovascular disease by gender. 
However, this problem did not hamper the present mortality forecasting, because the fitted values in the non-identifiable APC model were the same for all possible sets of parameter estimates.

In accordance with the WHO action plan, we calculated the population attributable fractions (PAF) and the probability of death in the absence of other causes of death for a partial elimination of exposure using the formulas presented in the online supplementary appendix 1 . Specifically, we considered a $30 \%$ reduction in the prevalence of smoking, a $25 \%$ reduction in the prevalence of hypertension, a $30 \%$ reduction in the prevalence of diabetes, and a 30\% reduction in the prevalence of hyperlipidaemia. All statistical analyses were performed using SAS software (9.4 version, SAS).

\section{RESULTS}

Figure 1 shows the age-adjusted mortality rates for heart disease, cerebrovascular disease and cardiovascular disease from 1990 to 2014 and the projections for 2015 to 2024 for men and women. For heart disease in men, 285 deaths per 100000 population occurred from 2010 to 2014 - a 1.12-fold
(A)

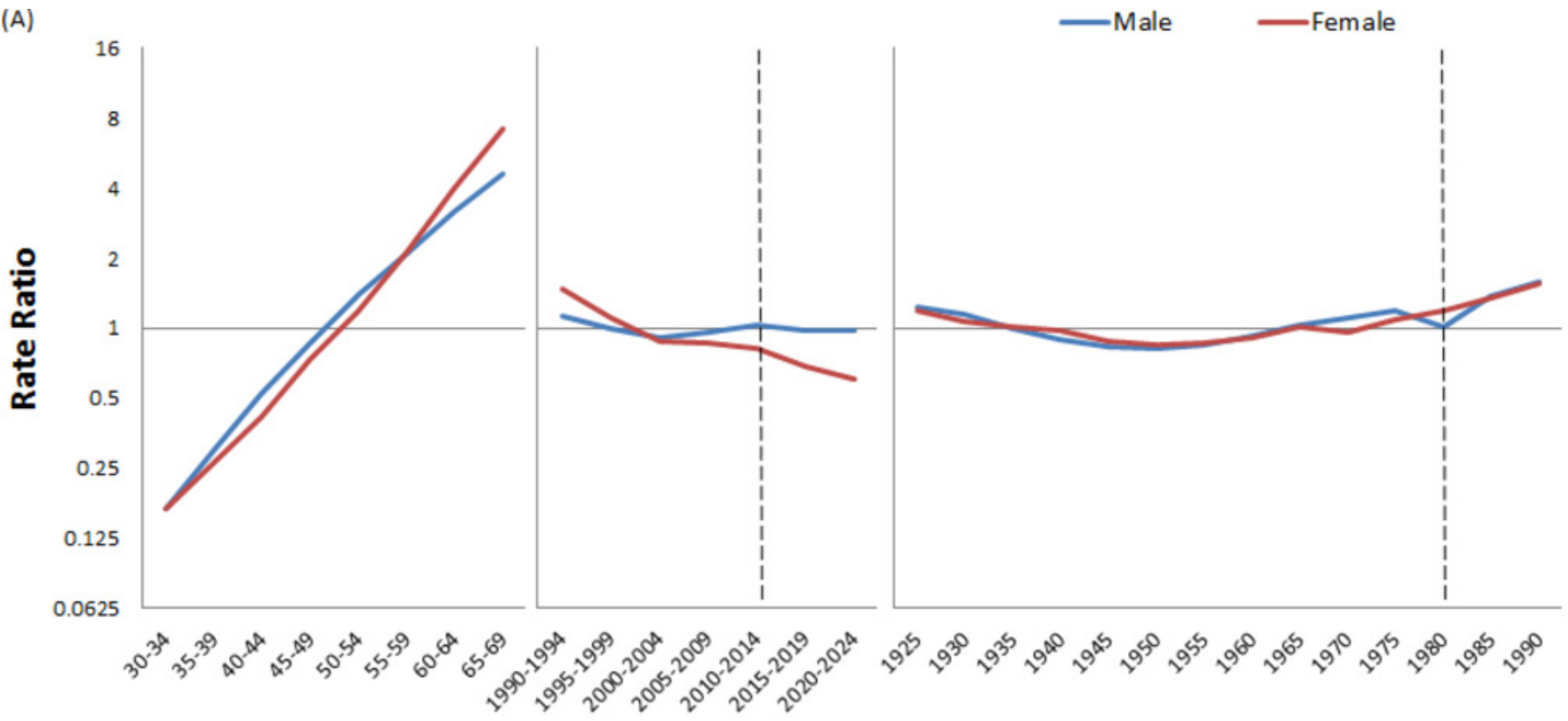

Period

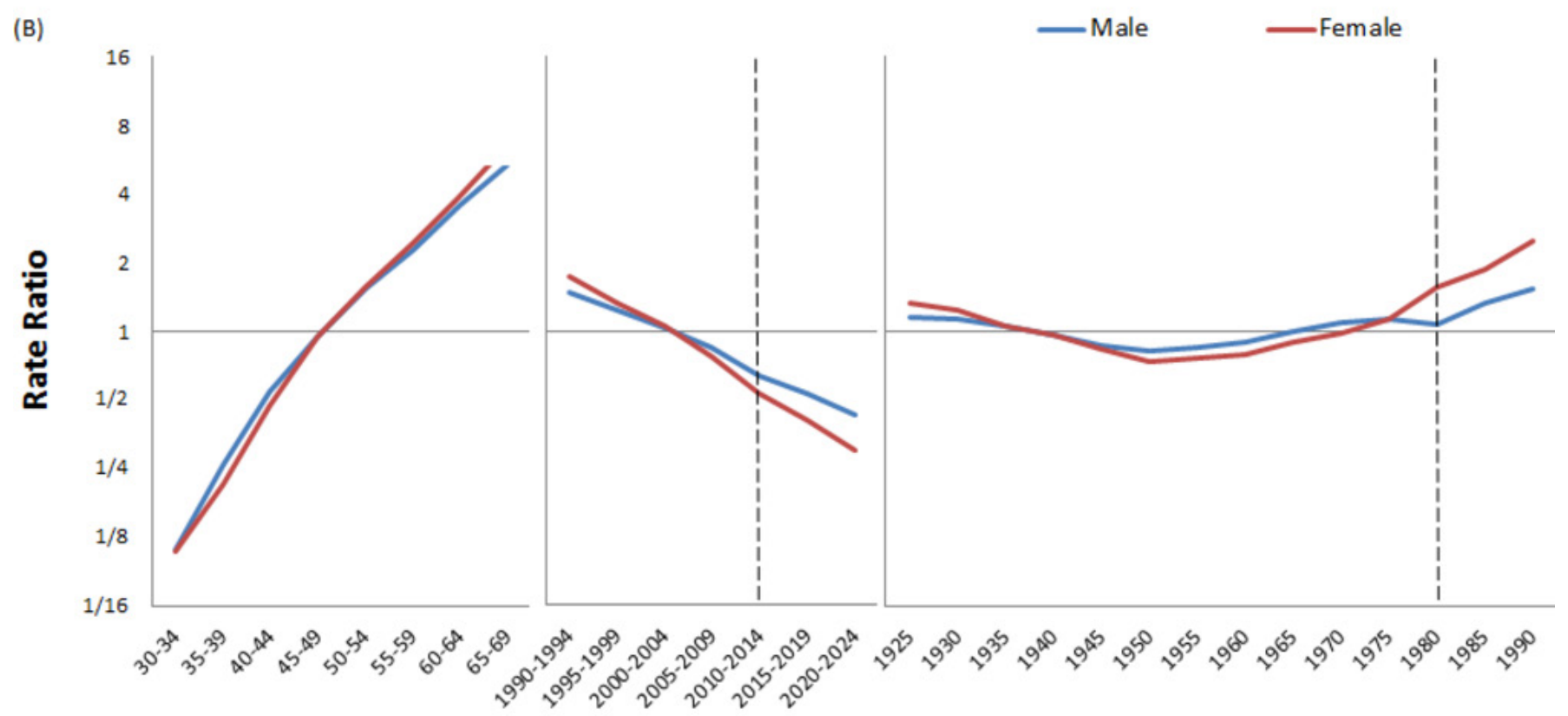

Age

Period

Cohort

Figure 2 Age, period, and cohort effects and their projected effects on heart disease (panel A) and cerebrovascular disease (panel B) among men (blue line) and women (red line). 
increase from 2000 to 2004 and a 0.79 -fold decrease from 1990 to 1994 . For heart disease in women, 86 deaths per 100000 population occurred from 2010 to 2014 - a 0.47 -fold decrease from 1990 to 1994 . The estimated projections for 2020-2024 for men and women were 303 and 69 deaths per 100000 population, respectively. The disease trends for cerebrovascular disease in men and women decreased by 0.28 -fold and 0.14-fold, respectively, from 1990 to 2024. For cardiovascular diseases, the age-adjusted mortality showed a decreasing trend for both sexes: 0.53 -fold for men and 0.24-fold for women from 1990 to 2024. In addition, the age-adjusted mortality rates for men were greater than the rates for women for both diseases, and the sex ratios (men/women) were progressively larger over the 25 years for both diseases. The sex ratios rose from 1.96 to 3.30 for heart disease between the periods of 1990-1994 and 2010-2014, and from 1.64 to 2.78 for cerebrovascular disease.

Figure 2 shows the APC analysis and the forecasting effects of heart disease (upper panel) and cerebrovascular disease (lower panel). For age effects in males, the mortality rate of the oldest 65-69 year age group was 28-fold that of the youngest 30-34 year age group for heart disease, and 49-fold for cerebrovascular disease. In comparison, the ratios of age effects for females were a little stronger. On the other hand, the trends in the period effect of heart disease and cerebrovascular disease in both sexes were similar to the age-adjusted mortality rates (figure 1) and the basic descriptive graphs by age and calendar year (see online supplementary figure S1). The forecasting periods of 2015-2019 and 2020-2024 were all smaller than 2010-2014 for both sexes in terms of heart disease and cerebrovascular disease. In terms of cohort effects, heart disease and cerebrovascular disease in both sexes showed a falling trend from 1925 to 1950 , and a rising trend thereafter. These trends were consistent with the association between westernisation and CVD at the developing stage after the 1970s. At this stage, those people born after the 1950 s faced the impact of westernisation, resulting in metabolic syndrome and obesity. The forecasting cohorts in 1985 and 1990 were all larger than that in 1980 for heart disease and cerebrovascular disease in both sexes.

Through the analysis and forecast by APC modelling, we combined heart disease and cerebrovascular disease as cardiovascular disease and used the probability of death to estimate the percent change between 2010-2014 and 2020-2024 (table 1). A total of 30103 deaths from cardiovascular diseases (19100 from heart disease and 11003 from cerebrovascular disease) occurred from 2010 to 2014 . The percent change in the business-as-usual trend between 2010-2014 and 2020-2024 would be a 6\% (range 1.7-10.1\%) lower risk of premature cardiovascular disease mortality among men. Even if each risk factor target were achieved, the percent change would still be far below the 25 by 25 goal for cardiovascular disease in men. Among men, the percent change in the smoking target was larger than that in the other risk factor targets, in that a $14.5 \%$ (range 10.6-18.3\%) reduction would save 3706 (3543-3868) men from premature death. The results for cerebrovascular disease in men were inconsistent, in that they showed a $26.3 \%(22.1-$ $30.2 \%)$ reduction in the business-as-usual trend and a $33.5 \%$ (29.8-37.0\%) reduction in the trend where the smoking target was achieved. A total of 1077 (1006-1155) men would be saved from death from cerebrovascular disease if the smoking target were reached. In contrast, the results for heart disease in men showed a $5 \%(9.7-0.5 \%)$ increment in the business-as-usual trend and a $4.8 \%(0.6-8.8 \%)$ reduction in the trend where the smoking target was achieved. A total of 2628 (2485-2782) men 
Table 2 Values of the population attributable fraction (PAF) for heart disease, cerebrovascular disease, and cardiovascular disease by gender and four risk factors in 2020-2024

\begin{tabular}{|c|c|c|c|c|c|}
\hline Gender & Outcome & $\begin{array}{l}30 \% \text { reduction in smoking } \\
\text { prevalence }\end{array}$ & $\begin{array}{l}25 \% \text { reduction in } \\
\text { hypertension prevalence }\end{array}$ & $\begin{array}{l}30 \% \text { reduction in diabetes } \\
\text { prevalence }\end{array}$ & $\begin{array}{l}30 \% \text { reduction in } \\
\text { hyperlipidaemia prevalence }\end{array}$ \\
\hline \multicolumn{6}{|l|}{ Men } \\
\hline & Heart disease & $10.4 \%$ (9.0 to 11.7$)$ & $3.0 \%(2.1$ to 3.9$)$ & $0.7 \%(0.4$ to 1.1$)$ & $3.5 \%$ (2.8 to 4.3$)$ \\
\hline & Cerebrovascular disease & $10.4 \%$ (6.3 to 14.0$)$ & $4.0 \%(3.6$ to 4.4$)$ & $0.6 \%(0.1$ to 1.1$)$ & $1.7 \%(1.3$ to 2.2$)$ \\
\hline & Cardiovascular disease & $10.4 \%$ (8.2 to 12.4$)$ & $3.3 \%(2.5$ to 4.0$)$ & $0.7 \%(0.3$ to 1.1$)$ & $3.0 \%(2.4$ to 3.7$)$ \\
\hline \multicolumn{6}{|l|}{ Women } \\
\hline & Heart disease & $1.6 \%(1.2$ to 2.0$)$ & $2.8 \%(2.1$ to 3.5$)$ & $0.7 \%(0.4$ to 1.0$)$ & $3.2 \%(2.7$ to 3.8$)$ \\
\hline & Cerebrovascular disease & $1.6 \%(1.1$ to 2.3$)$ & $3.5 \%(3.2$ to 3.9$)$ & $0.5 \%(0.2$ to 1.0$)$ & $1.6 \%(1.1$ to 2.0$)$ \\
\hline & Cardiovascular disease & $1.6 \%(1.2$ to 2.1$)$ & $3.1 \%(2.5$ to 3.6$)$ & $0.7 \%(0.3$ to 1.0$)$ & $2.7 \%$ (2.1 to 3.2 ) \\
\hline
\end{tabular}

would be saved from death from heart disease if the smoking target were reached. Even if no risk factor targets were achieved, the results for cardiovascular disease in women all showed more than a $25 \%$ reduction before 2025 . Among women, achieving the smoking target could help reduce deaths by $29.9 \%$ (23.9$35.3 \%)$. That was a little larger than the other risk factors, and would save 159 (145-173) women from premature death. The probability of death from cerebrovascular disease could be reduced by $37.5 \%(31.4-43.0 \%)$ in the business-as-usual trend and by $39.7 \%(33.8-44.9 \%)$ in the trend where the smoking target was achieved. In terms of heart disease among women, the percent change was only a $21.5 \%(15.0-27.4 \%)$ lower risk in the business-as-usual trend.

The PAF values of the four risk factors for both genders are shown in table 2 . The PAF values for the smoking target were larger than the other risk factors in men. A 10.4\% (8.2-12.4\%) reduction in cardiovascular disease mortality would occur if the smoking prevalence decreased by $30 \%$. Similarly, $10.4 \%$ $(6.3-14.0 \%)$ and $10.4 \%(9.0-11.7 \%)$ reductions in cerebrovascular disease and heart disease, respectively, would occur if the smoking prevalence decreased by $30 \%$. The PAF values for the other risk factors in men were all $\leq 4 \%$. On the other hand, the PAF values were all $<4 \%$ in women.

To understand more clearly why the premature cardiovascular disease mortality could not be reduced by $25 \%$ before 2025 among men by simply reaching the risk factor targets, we determined the PAF values by different age groups with the corresponding number of persons who would be saved from death if the risk factor targets were achieved (figure 3). For the smoking target, we found that the PAF values decreased from the youngest age group (17\%) to the oldest age group (5\%), but the corresponding number of persons who would be saved from deaths was inconsistent with the PAF values. A total of 108 people would be saved from death in the 30-34 year age group, and 740 in the 60-64 year age group. This result might be reasonable because the disease rates between 30 and 44 were smaller than the rates in the older age groups. Furthermore, this result might also help us to find out which age group was more effective for the control of cardiovascular disease. Despite the PAF values between 30 - and 44-year-olds being higher, the number of persons who would be saved from deaths were still small. It might indicate that the contribution in the younger people would be limited even if the department of health promoted the stringent control of smoking. The same situation also happened in the 65-69 year age group because the PAF value was smaller.

We used the stringent control of smoking to estimate the percent change and the corresponding number of men who would be saved from death from cardiovascular disease (table 3). A 50\% reduction in the prevalence of smoking would decrease deaths by $20.3 \%$ (16.7-23.8\%) and save 6176 (5906$6444)$ men from death due to cardiovascular disease, and would decrease deaths by $11.4 \%(7.5-15.1 \%)$ and save 4381 (41414637) men from premature death caused by heart disease. The more stringent control of a $70 \%$ reduction in the prevalence of smoking would decrease deaths by $26.2 \%(22.9-29.3 \%)$ and save 8646 (8271-9025) men from death due to cardiovascular disease, and would decrease deaths by $18.1 \%(14.5-21.4 \%)$ and save 6133 (5798-6492) men from premature death caused by heart disease.

\section{DISCUSSION}

Our study showed that the reduction of premature mortality in females with cardiovascular disease may reach or exceed $25 \%$ before 2025 even if no targeted risk factors (smoking, hypertension, diabetes and hyperlipidaemia) were achieved. For males with cardiovascular disease, the inhibition of smoking behaviour would be more effective and important than the control of hypertension, diabetes, and hyperlipidaemia, but the 25 by 25 goal would still not be reached. To achieve the 25 by 25 goal, the stringent control of smoking, such as a $70 \%$ reduction in prevalence, would be necessary. Controlling smoking in the 45-64 year age group may be the most effective way to prevent cardiovascular disease.

Although the number of deaths from premature cardiovascular disease in women can probably reach the 25 by 25 goal without any control of the risk factors, the same is not true for men. This result was not similar to that of Sacco and colleagues who estimated the 25 by 25 goals for mortality from premature cardiovascular diseases in specific regions of the world. ${ }^{23}$ They showed that the probability cannot decrease by $25 \%$ before 2025 in specific regions of Eastern Europe and Central Asia, sub-Saharan Africa, North Africa and the Middle East, South Asia, East Asia and the Pacific, and Latin America and the Caribbean; however, the estimates of future trends in premature cardiovascular diseases show substantial variation across countries. Although their results across all regions were not similar to our results for both sexes, the pattern in high-income countries was consistent only among women. This indicated that comprehensive public health, medical care and treatment are effective in preventing future premature deaths from cardiovascular diseases in high-income countries. For example, the government of Taiwan implemented a National Health Insurance programme in 1995, and almost every person now has access to preventive healthcare and medical treatment. From 1982 to 1995 life expectancy increased by 2.43 years and mortality decreased by $17.2 \%$; these figures improved to 4.01 years and $25.2 \%$, respectively, from 1995 to 2008 . 
$30 \%$ Smoking reduction

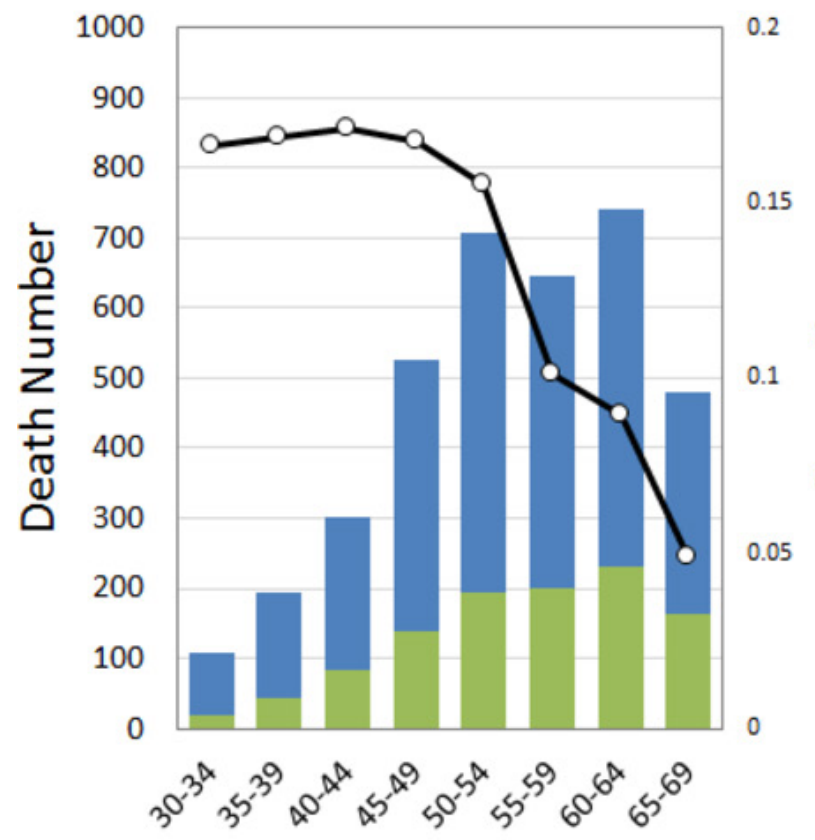

Age

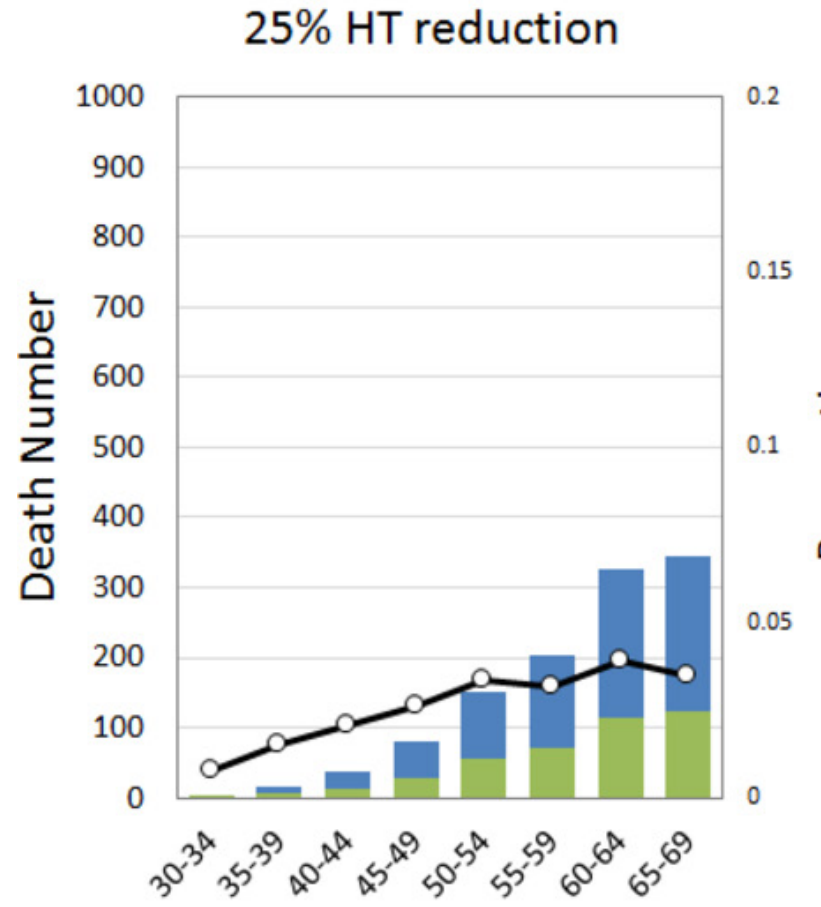

Age
$30 \%$ DM reduction

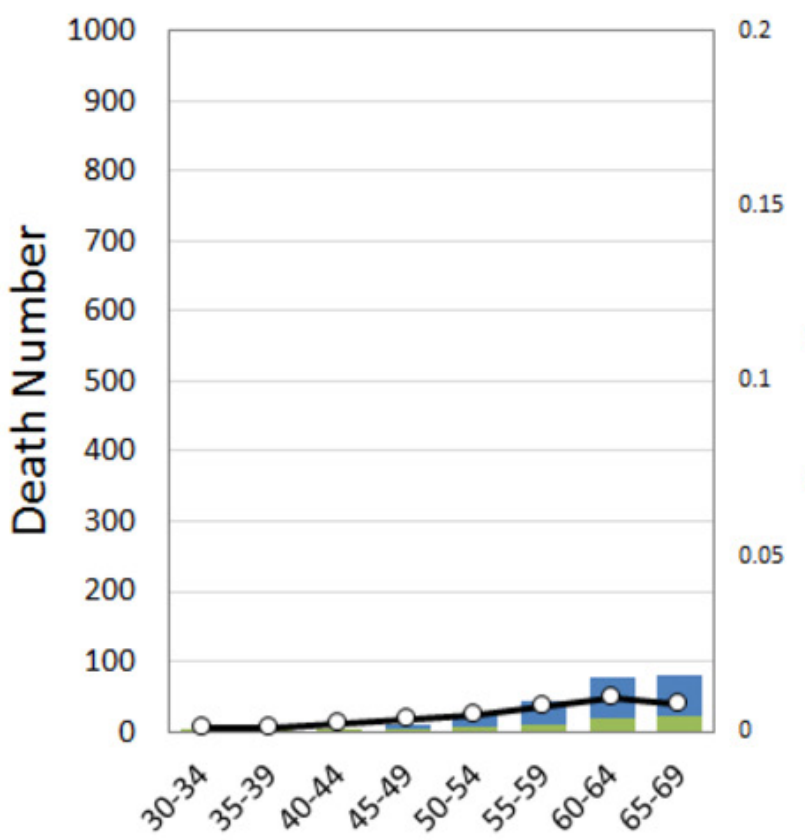

Age

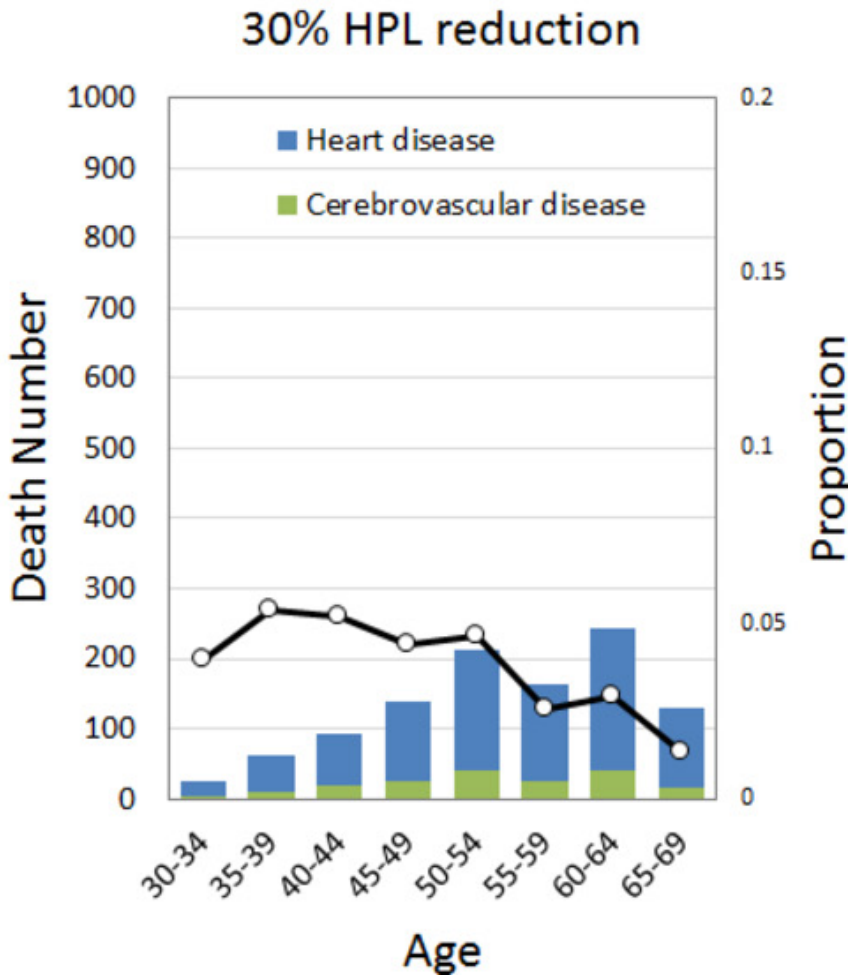

Age

Figure 3 Number of persons who would be saved from death if the risk factor targets for specific age groups and the corresponding values for population attributable fractions of cardiovascular diseases (heart disease plus cerebrovascular disease) among men were reached. DM, diabetes mellitus; HPL, hyperlipidaemia; HT, hypertension.

Epidemiological transition might explain the mortality pattern in premature cardiovascular diseases in Taiwan and may be a possible reason for the difficulty in reaching the 25 by 25 goal for men. In general, epidemiological transition describes the changing process of demographic and health-related factors such as population growth, ageing, morbidity, and mortality. ${ }^{24}$ Although the mortality pattern of cardiovascular diseases in most countries cannot be fully explained by epidemiological transition, the dramatic decreasing trend of cardiovascular diseases in high-income countries can still be largely explained by it. ${ }^{122526}$ For example, a study by Santosa and colleagues indicated that the 25 by 25 goal will be difficult 
Table 3 Percent changes in the probability of death from heart disease, cerebrovascular disease, and cardiovascular disease after the stringent control of smoking in men

\begin{tabular}{|c|c|c|c|c|}
\hline Outcome & $\begin{array}{l}\text { Percent change if a } 50 \% \\
\text { reduction in smoking } \\
\text { prevalence was reached }\end{array}$ & $\begin{array}{l}\text { Men who would be saved } \\
\text { from death if a } 50 \% \text { reduction } \\
\text { in smoking prevalence was } \\
\text { reached }\end{array}$ & $\begin{array}{l}\text { Percent change if a } 70 \% \\
\text { reduction in smoking } \\
\text { prevalence was reached }\end{array}$ & $\begin{array}{l}\text { Men who would be saved } \\
\text { from death if a } 70 \% \text { reduction } \\
\text { in smoking prevalence was } \\
\text { reached }\end{array}$ \\
\hline Heart disease & $-11.4 \%(-7.5$ to -15.1$)$ & 4381 (4141 to 4637 ) & $-18.1 \%(-14.5$ to -21.4$)$ & 6133 (5798 to 6492) \\
\hline Cerebrovascular disease & $-38.3 \%(-34.9$ to -41.5$)$ & 1795 (1677 to 1925$)$ & $-43.2 \%(-40.1$ to -46.1$)$ & 2513 (2347 to 2696) \\
\hline Cardiovascular disease & $-20.3 \%(-16.7$ to -23.8$)$ & 6176 (5906 to 6444) & $-26.2 \%(-22.9$ to -29.3$)$ & 8646 (8271 to 9025$)$ \\
\hline
\end{tabular}

to reach in Sweden because they are in a late stage of epidemiological transition, and the probability of premature death from cardiovascular disease decreased by $48.3 \%$ from 1991 to $2006 .{ }^{13}$ In our findings, mortality from premature cardiovascular disease in women and men decreased by $66 \%$ and $42 \%$, respectively, from 1990 to 2014, and the mortality pattern was stable after 2014 . This is consistent with the pattern in the late stage of epidemiological transition. ${ }^{27}$

Following the disease guidelines of the Ministry of Health and Welfare in Taiwan, cardiovascular disease can be categorised as heart disease, cerebrovascular disease and hypertensive disease. Nevertheless, we excluded hypertensive disease in this study design in order to avoid biased results. Based on the definition of this disease, hypertensive disease includes hypertension and its complications, and the risk factors targeted in the 25 by 25 goal included the control of hypertension. If we included hypertensive disease in the statistical analysis, the target hypertension (a $25 \%$ reduction in prevalence) would lead to a $25 \%$ reduction in hypertensive disease mortality.

We excluded physical activity in our study design because previous investigations in Taiwan were not appropriate. Most used self-assessment questionnaires and the levels and frequency of physical activity were subjective. They were also too limited by recall bias to reflect the long-term distribution of physical activity in the population. ${ }^{28}$ Therefore, we turned to an investigation of blood lipids because the criteria for hyperlipidaemia were objective and more comparable than the criteria for the amount of exercise. We also excluded alcohol and salt/ sodium because previous studies were inadequate. The online database of long-term surveillance by the Nutrition and Health Survey in Taiwan was a possible choice for the population distributions of alcohol and salt/sodium intake. This surveillance included seven investigations since 1980, but the last two were not national in scope (one involving junior high school students in 2010 and the other of senior high school students in 2011). The most recent investigation of national scope was from 2005 to 2008. Nevertheless, this investigation was inadequate and did not reflect the population distribution after 2009 because of the large variations in the population distribution, dietary patterns, and nutritional intake. ${ }^{29-32}$

We estimated the contributions of the four risk factors by the PAF method as was done in previous studies. ${ }^{14} 33$ Kontis and colleagues used a time-based population impact fraction (PIF), which is an extension of the PAF method, but they let the prevalence and relative risk of risk factors depend on the calendar year; Lim and colleagues used an ordinary PAF. Despite these differences, the results were similar, especially for the estimation of NCDs attributable to risk factors in men. The control of smoking was an effective way to prevent NCDs and cardiovascular disease.

Our study demonstrated the contributions of targeted risk factors, but there were limitations. First, we used the prevalence of hyperlipidaemia in 2009 to represent the population distribution in Taiwan. This assumed that the prevalence for each age group in 2009 would not be much different from the prevalence after 2009. This assumption is inappropriate, but the consequences are allowable because of the limited database. The investigation by the National Health Interview Survey in 2009 was the latest database which measured the prevalence of hyperlipidaemia. An earlier investigation was the Taiwan Nationwide Population-based Prevalence Survey of High Blood Pressure, Hyperglycemia, and High Blood Lipid Profile (usually abbreviated as Triple-High status) in 2002, and its follow-up survey in 2007. From the reports about Triple-High status in 2002 and 2007, the 5-year change in high blood lipids was not very different. $^{21}$

Second, we evaluated the contributions of single risk factors without further analysis of the contribution of multiple risk factors. Unfortunately, there was no available database which could be used. It might also be noted that middle and low income countries may be limited in terms of databases. Therefore, the analysis of single risk factors makes more sense for these countries. Finally, we chose a 5-year calculation for APC analysis in forecasting mortality to replace the single year calculation, and this was different from previous studies. ${ }^{13}{ }^{14}$ This avoided a potential problem of forecasting error. The more points in time that are forecast, the greater the likelihood of errors. A total of 11 points in time are needed to forecast from 2014 to 2025 by single year calculations, but only two points by 5 -year calculations. Therefore, the APC forecasting analysis was an appropriate method.

Although we believe that our study design and analysis were appropriate, there are some points worth considering in order to improve further research. Lloyd-Sherlock and colleagues argued that the 25 by 25 goal proposed by WHO and the UN is incompatible with the present needs of an ageing population, and indicated that because of institutional ageism, the survival of younger groups is more important than survival after age $70 .^{34}$ According to cancer statistics in 2015 for Taiwan, 21397 people who were more than 70 years old died of cardiovascular disease; that was $70 \%$ of the total deaths from cardiovascular disease. Therefore, we suggest that older people should be included in further research. Additionally, Bonita and Beaglehole argued that the 25 by 25 goal is insufficient for Asian women because secondhand smoke may be a factor for them, and the effect of a $30 \%$ reduction in tobacco use may be limited. ${ }^{35} 36$ Prevention of exposure to secondhand smoking should be considered as a component of the smoking target for woman.

\section{CONCLUSIONS}

Our study developed a process to evaluate the contribution of risk factors related to NCDs, and the results provided an important reference to improve the rates of mortality from 
NCDs in the future. Our results suggested that smoking was the primary risk factor in the prevention of cardiovascular disease, and stringent control of cigarette smoking can help to achieve the 25 by 25 goal.

\section{Key messages}

\section{What is already known about this subject?}

Cardiovascular diseases (heart disease plus cerebrovascular disease) have been the second and third leading causes of death in Taiwan since 1997. The 25 by 25 goal proposed by WHO was important in establishing relevant healthy policy, but no previous study provided reference data in Taiwan.

\section{What might this study add?}

We added the risk factor of hyperlipidaemia as a target in estimating the 25 by 25 goal in Taiwan. The trend of premature mortality in female cardiovascular disease can reach or exceed a $25 \%$ reduction before 2025 without any control of this risk factor. Smoking is the primary risk factor for premature cardiovascular mortality among men rather than the other risk factors of hypertension, diabetes, and hyperlipidaemia.

\section{How might this impact clinical practice?}

Smoking cessation in the 45-64 year age group may lessen the risk of cardiovascular disease. The stringent control of cigarette smoking such as a 70\% reduction in its prevalence can help Taiwan reach the $25 \%$ reduction in premature cardiovascular mortality in males before 2025 .

\section{Author affiliations}

${ }^{1}$ Institute of Epidemiology and Preventive Medicine, College of Public Health, National Taiwan University, Taipei, Taiwan

${ }^{2}$ Research Center for Genes, Environment and Human Health, College of Public Health, National Taiwan University, Taipei, Taiwan

${ }^{3}$ Department of Internal Medicine, Division of Chest Medicine, National Taiwan University Hospital, Taipei, Taiwan

${ }^{4}$ Department of Internal Medicine, School of Medicine, National Taiwan University, Taipei, Taiwan

${ }^{5}$ Department of Internal Medicine and Cardiovascular Center, National Taiwan University Hospital, Taipei, Taiwan

${ }^{6}$ Institute of Occupational Medicine and Industrial Hygiene, National Taiwan University College of Public Health, Taipei, Taiwan

${ }^{7}$ Stroke Center \& Department of Neurology, National Taiwan University Hospital, Taipei, Taiwan

${ }^{8}$ Department of Internal Medicine, National Taiwan University Hospital, Taipei, Taiwan

Contributors SYS was in charge of data collection, analysis, and wrote the first draft and the revision of the manuscript. WCL and KLC contributed to study design and wrote the part of first draft of the manuscript. TTC was in charge of data collection. All authors approved the final version of the manuscript.

Funding The study was supported by the Ministry of Science and Technology, Taiwan, (MOST 104-2321-B-002-077 -, MOST 103-2314-B-002 -135 -MY3).

\section{Competing interests None declared.}

Ethics approval The proposal was approved by the IRB, National Taiwan University, and no consent from participants was needed because of anonymity.

Provenance and peer review Not commissioned; externally peer reviewed.

(c) Article author(s) (or their employer(s) unless otherwise stated in the text of the article) 2017. All rights reserved. No commercial use is permitted unless otherwise expressly granted.

\section{REFERENCES}

1. Lozano R, Naghavi M, Foreman K, et al. Global and regional mortality from 235 causes of death for 20 age groups in 1990 and 2010: a systematic analysis for the Global Burden of Disease study 2010. Lancet 2012;380:2095-128.

2. Alwan $A$, Maclean DR, Riley LM, et al. Monitoring and surveillance of chronic noncommunicable diseases: progress and capacity in high-burden countries. Lancet 2010;376:1861-8
3. Zoghbi WA, Duncan T, Antman E, et al. Sustainable development goals and the future of cardiovascular health: a statement from the global Cardiovascular Disease Taskforce. J Am Heart Assoc 2014;3:e000504.

4. Beaglehole R, Bonita R, Alleyne G, et al. UN high-level meeting on non-communicable diseases: addressing four questions. Lancet 2011;378:449-55.

5. World Health Organization. Global action plan for the prevention and control of noncommunicable diseases 2013-2020, 2013.

6. Grainger-Gasser A, Perel P, Lagier-Hässig L, et al. The Road to $25 \times 25$ : update on WHF CVD Roadmaps. Glob Heart 2016.

7. Guwatudde D, Mutungi G, Wesonga R, et al. The epidemiology of hypertension in Uganda: findings from the National Non-Communicable Diseases Risk Factor Survey. PLoS One 2015;10:e0138991.

8. Ebrahim S, Pearce N, Smeeth $\mathrm{L}$, et al. Tackling non-communicable diseases in low- and middle-income countries: is the evidence from high-income countries all we need? PLoS Med 2013:10:e1001377.

9. Reddy KS, Hunter DJ. Noncommunicable diseases. N Eng/ J Med 2013;369:2563.

10. Hogerzeil HV, Liberman J, Wirtz VJ, et al. Promotion of access to essential medicines for non-communicable diseases: practical implications of the UN political declaration. Lancet 2013;381:680-9.

11. Bonita R, Magnusson R, Bovet $P$, et al. Country actions to meet UN commitments on non-communicable diseases: a stepwise approach. Lancet 2013;381:575-84.

12. Roth $G A$, Forouzanfar $M H$, Moran $A E$, et al. Demographic and epidemiologic drivers of global cardiovascular mortality. N Engl J Med 2015;372:1333-41.

13. Santosa A, Rocklöv J, Högberg U, et al. Achieving a $25 \%$ reduction in premature noncommunicable disease mortality: the Swedish population as a cohort study. BMC Med 2015:13:65.

14. Kontis V, Mathers CD, Rehm J, et al. Contribution of six risk factors to achieving the $25 \times 25$ non-communicable disease mortality reduction target: a modelling study. Lancet 2014;384:427-37.

15. Wagner KH, Brath H. A global view on the development of non communicable diseases. Prev Med 2012;54(Suppl):S38-41.

16. 2016 Statistics of causes of death. 7 Sep ed: Ministry of Health and Welfare, 2016.

17. Singh GM, Danaei G, Farzadfar F, et al. The age-specific quantitative effects of metabolic risk factors on cardiovascular diseases and diabetes: a pooled analysis. PLoS One 2013:8:e65174.

18. Wang J, Thornton JC, Russell M, et al. Asians have lower body mass index (BMI) but higher percent body fat than do whites: comparisons of anthropometric measurements. Am J Clin Nutr 1994;60:23-8.

19. Romero-Corral A, Somers VK, Sierra-Johnson J, et al. Accuracy of body mass index in diagnosing obesity in the adult general population. Int J Obes 2008;32:959-66.

20. Dudeja V, Misra A, Pandey RM, et al. BMI does not accurately predict overweight in Asian Indians in northern India. Br J Nutr 2001;86:105-12.

21. Huang HL, Yen YY, Lin PL, et al. Household secondhand smoke exposure of elementary schoolchildren in Southern Taiwan and factors associated with their confidence in avoiding exposure: a cross-sectional study. BMC Public Health 2012;12:40.

22. Tzeng IS, Lee WC. Forecasting hepatocellular carcinoma mortality in Taiwan using an age-period-cohort model. Asia Pac J Public Health 2015;27:NP65-73.

23. Sacco RL, Roth GA, Reddy KS, et al. The Heart of 25 by 25 : achieving the goal of reducing global and regional premature deaths from cardiovascular diseases and stroke: a modeling study from the American Heart Association and World Heart Federation. Circulation 2016;133:e674-90.

24. McKeown RE. The epidemiologic transition: changing patterns of mortality and population dynamics. Am J Lifestyle Med 2009;3(1 Suppl):19S-26.

25. Yusuf S, Reddy S, Ounpuu S, et al. Global burden of cardiovascular diseases: part II: variations in cardiovascular disease by specific ethnic groups and geographic regions and prevention strategies. Circulation 2001;104:2855-64.

26. Reddy KS, Yusuf S. Emerging epidemic of cardiovascular disease in developing countries. Circulation 1998:97:596-601.

27. Santosa A, Wall S, Fottrell E, et al. The development and experience of epidemiological transition theory over four decades: a systematic review. Glob Health Action 2014;7:23574

28. Sallis JF, Saelens BE. Assessment of physical activity by self-report: status, limitations, and future directions. Res Q Exerc Sport 2000;71(Suppl 2):1-14.

29. Popkin BM. The nutrition transition: an overview of world patterns of change. Nutr Rev 2004:62(7 Pt 2):S140-3.

30. Popkin BM. The nutrition transition and obesity in the developing world. J Nutr 2001;131:871S-3.

31. Popkin BM. The nutrition transition in low-income countries: an emerging crisis. Nutr Rev 1994;52:285-98.

32. Popkin BM. Global nutrition dynamics: the world is shifting rapidly toward a diet linked with noncommunicable diseases. Am J Clin Nutr 2006;84:289-98.

33. Lim SS, Vos T, Flaxman AD, et al. A comparative risk assessment of burden of disease and injury attributable to 67 risk factors and risk factor clusters in 21 regions, 19902010: a systematic analysis for the Global Burden of Disease study 2010. Lancet 2012;380:2224-60.

34. Lloyd-Sherlock PG, Ebrahim S, McKee M, et al. Institutional ageism in global health policy. BMJ 2016;354:i4514. 
35. Bonita R, Beaglehole R. Women and NCDs: overcoming the neglect. Glob Health Action 2014;7:23742.
36. Oberg M, Jaakkola MS, Woodward A, et al. Worldwide burden of disease from exposure to second-hand smoke: a retrospective analysis of data from 192 countries. Lancet 2011;377:139-46. 